\title{
UTILIZATION OF PHOSWICH DETECTORS FOR SIMULTANEOUS, MULTIPLE RADIATION DETECTION.
}

\author{
by \\ William H. Miller \\ Manuel Diaz de Leon \\ Nuclear Science and Engineering Institute \\ University of Missouri-Columbia \\ Columbia, MO 65211, USA \\ 573-882-9692 \\ MillerW@missouri.edu
}

\author{
Presented to \\ Methods and Applications of Radioanalytical Chemsitry \\ MARC VI \\ (Accepted for Publication)
}

April $7-112003$ 
Utilization of Phoswich Detectors for Simultaneous, Multiple Radiation Detection, W.H.Miller and M.Diaz de Leon, Nuclear Science and Engineering Institute, University of Missouri-Columbia, Columbia, MO 65211, USA

\begin{abstract}
:
A phoswich radiation detector is comprised of a phosphor sandwich in which several different phosphors are viewed by a common photomultiplier. By selecting the appropriate phosphors, this system can be used to simultaneously measure multiple radiation types (alpha, beta, gamma and/or neutron) with a single detector. Differentiation between the signals from the different phosphors is accomplished using digital pulse shape discrimination techniques. This method has been shown to result in accurate discrimination with highly reliable and versatile digital systems. This system also requires minimal component count (i.e. only the detector and a computer for signal processing).

A variety of detectors of this type have been built and tested including: 1) a triple phoswich system for alpha/beta/gamma swipe counting, 2) two well-type detectors for measuring low levels of low energy photons in the presence of a high energy background, 3) a large area detector for measuring beta contamination in the presence of a photon background, 4) another large area detector for measuring low energy photons from radioactive elements such as uranium in the presence of a photon background. An annular geometry, triple phoswich system optimized for measuring alpha/beta/gamma radiation in liquid waste processing streams is currently being designed.
\end{abstract}

\title{
Phoswich Detector Overview:
}

A phoswich detector is a scintillation detector system which consists of two or more different scintillators coupled to a single photomultiplier tube. Phosphors are selected such that each will be sensitive to only one particular type of radiation (i.e., alpha, beta, gamma and/or neutron). Pulses created by the photomultiplier tube have characteristics (rise times) that are directly related to the decay fluorescence time for each of the individual phosphors which can be electronically differentiated. This discrimination has been previously implemented using analog pulse shaping techniques, but recent work by these authors has focused on digital pulse shape discrimination. A typical detector design is shown in Figure 1.

The ability to discriminate between different radiation particles by pulse shape discrimination (PSD) has been known for some time. Owen ${ }^{1}$ published an article in 1962, "Pulse Shape Discrimination - A Survey of Current Techniques," that outlined the differences in pulses from different scintillators. He discussed alpha and electron (gamma-ray induced or primary beta particles) discrimination in $\mathrm{Nal}(\mathrm{Tl})$; alpha, proton, and electron (gamma-ray induced or beta) discrimination in $\mathrm{CsI}(\mathrm{TI})$; alpha, electron (gamma-ray), and proton (neutron induced) discrimination in stilbene and other organic scintillators; and proton and alpha discrimination in $\mathrm{ZnS}(\mathrm{Ag})$. 
Practical, commercial phoswich systems, such as the Harshaw $\mathrm{CsF}_{2} / \mathrm{Nal}$ phoswich TASC12 detector $^{2}$, were initially produced in the 1970s for swipe counting. This phoswich detector consisted of an optically coupled sandwich of $\mathrm{CaF}_{2}(\mathrm{Eu})$ and $\mathrm{Nal}(\mathrm{TI}) . \mathrm{CaF}_{2}(\mathrm{Eu})$ is used to detect charged particles and has a decay constant of $0.9 \mu \mathrm{sec}$. $\mathrm{Nal}(\mathrm{TI})$ is used for gamma-ray detection and has a decay constant of $0.23 \mu \mathrm{sec}$. A combination of pulse shape and pulse height analysis allows this detector to discriminate between alpha, beta and gamma-ray radiation. It was found that this system worked well, but the analog, pulse shape discrimination circuitry had a large component count (many NIMBIN-based analog modules) and was found to be somewhat difficult to use with respect to stability and reliability.

Other phoswich systems have been developed over the years for assessing human exposure to radionuclides from uranium mill tailings ${ }^{3}$, measuring $L \mathrm{X}$-rays $I n$-vivo with a $\mathrm{Si}(\mathrm{TI})$ $\mathrm{Nal}(\mathrm{TI})$ detector ${ }^{4}$, determining $\mathrm{Sr}-90$ in radioactive waste using a low-level background counting system $^{5}$, simultaneously counting alpha, beta and thermal neutrons with $\mathrm{ZnS}(\mathrm{Ag})$, Li-glass and NE102A phoswich ${ }^{6}$ and measuring neutrons and charged particles in space applications ${ }^{7}$. This list is not meant to be comprehensive, but gives an indication of practical applications.

\section{Digital Pulse Shape Discrimination:}

Digital systems are rapidly displacing many analog circuits traditionally used in electronic systems. This is also occurring in radiation detection systems where significant research has taken place and new products based upon digital signal processing (DSP) technology are on the market. Analog-toDigital Conversion (ADC) technology has progressed to the point where it is now possible to digitize (e.g. at $100 \mathrm{MHZ}$ with 12-bit accuracy) the complete waveform of a pulse from a radiation detector and analyze it digitally, rather than capturing just the height of the pulse as is traditionally done. Figure 2 shows digitally sampled pulses from the Harshaw $\mathrm{CsF}_{2} / \mathrm{Nal}$ phoswich TASC-12 detector described above. This captured signal can then be used to correct for ballistic defect (changes in pulse characteristics due to variations in the location of radiation interaction in the detector) in intrinsic germanium detectors, eliminate noise induced or "noisy" pulses, validate a pulse, or allow for pulse shape discriminate as is needed for phoswich detectors.

The authors of this paper have developed several implementations of a digital signal processing system for radiation detectors. The first implementation was designed and fabricated with discrete components on a dedicated circuit board coupled to a PC to analyze a desktop version of the system. It utilized an Analog Devices 9020, 10 bit, 60 MHZ flash ADC, an IDT 7225A FIFO buffer, and a PC. It was capable of pulse shape discrimination and radiation spectroscopy at a rate of approximately 1000 counts per second. ${ }^{8}$ A second system utilized the same data acquisition components coupled to a TMS320C25 DSP operating at $40 \mathrm{MHZ}$. It demonstrated the capability of implementing this process in portable instrumentation because of the small size and low cost of the DSP chip. The current version uses a PCbased digital oscilloscope board (GaGeScope CompuScope 1012) ${ }^{9}$ with software developed specifically 
for this application. Current GaGeScope/personal computer systems are capable of pulse processing above 10,000 pulses per second.

Coupling the features of a phoswich detector to a digital pulse shape analysis system offers several advantages. First, the component count is reduced. Analog systems such as the Harshaw swipe counter $^{2}$ required several NIMBIN components (pulse shaping amplifiers, a pulse shape discrimination module, SCAs, counters, etc.) in addition to the detector module. The digital system requires only the detector module connected directly to a digitizer in a computer for simultaneously measuring several different types of radiation. Analog circuitry is minimized since the pulses are sent directly to the digitizer and no analog components are required for pulse shape analysis. A single computer provides all necessary interfacing for both detector modules and for system integration. Digital pulse shape analysis also provides other features that are not normally available in detector systems. By capturing the waveform of each incoming pulse the system can detect baseline drift, identify excessive noise or ripple due to failing components, reject noisy and noise induced pulses and eliminate over-voltage, saturated pulses. Algorithms to take advantage of these features can be easily incorporated into the software. The computer system can provide both the necessary front end software to acquire and process pulses from the detector module and a user friendly interface for operator interaction, graphical display, data analysis and storage, alarms, system calibration and system status.

To implement these features, software has been written with the following functions. First, the software dynamically changes the oscilloscope card's threshold to determine the minimum detectable energy pulse without continuous triggering. Following initialization, the system enters a pulse shape calibration routine where pulses are sampled and separated into distinct pulse shapes representing each of the types of radiation being detected. For the three phosphor detector system shown in Figure 1 this classification is for alpha particles, beta particles, and gamma-rays. Reference pulses for the different types of radiation are built from this information. Actual data acquisition then begins by checking each pulse using a series of tests to ensure that it is indeed a valid pulse. The first test calculates a constant baseline preceding the pulse to eliminate pulses involved in pulse pileup and to check for excessive noise in the baseline. The pulse is then digitally integrated and the baseline is subtracted to account for any $D C$ offset. The rise time of the pulse is then determined, representing the time from the beginning of the pulse to its peak to determine if it is in the expected range. Tests also reject pulses that saturate the detector (summation peaks and cosmic ray interactions) and those that would be off the scale of the energy spectrum displayed by the program. Once the pulses are validated, each pulse is examined for classification by radiation type. A cross-correlation algorithm is used to classify each pulse as to alpha, beta or gamma origination. Final corrections involve adjusting known biases in the detection system, i.e. the misclassification of gamma-ray interactions in a beta scintillator which would be recorded as beta particles. This correction is made by estimating a gamma spectrum in the beta crystal from the gamma spectrum seen in the gamma phosphor and subtracting it from the beta spectrum in the beta phosphor. 


\section{Fabricated Phoswich Detector Systems:}

To test various phoswich detection designs for a variety of applications, six different phoswich detectors have been built and one is currently under design. Most of these have also been tested with the GaGe-based digital pulse shape discrimination system described above.

First, the digital system was used to replace the analog system previously coupled to a Harshaw Csl/Nal phoswich TASC-12 detector for alpha/beta/gamma swipe counting. Table 1 gives results for this system in terms of the cross-talk (i.e., the percentage of events misclassified by the detector). Good performance of the digital system was achieved. ${ }^{10-11}$

Second, the digital system was used with a new, triple phosphor system. ${ }^{12}$ Discrimination results presented in Table 2 indicate excellent pulse shape discrimination, in particular, better discrimination between alpha and beta induced events as compared to the two phosphor system.

Two detectors for biological/medical applications have been fabricated and evaluated. Both are designed to detect low levels of low energy photons. One consists of a $2 \mathrm{~mm}$ thick $\mathrm{Csl}(\mathrm{Na})$ well detector inside of a $38.1 \mathrm{~mm} \times 12.7 \mathrm{~mm}$ well in a $76.2 \mathrm{~mm} \times 76.2 \mathrm{~mm} \mathrm{Nal}(\mathrm{TI})$ detector. The second is a $2 \mathrm{~mm} \times$ $12.7 \mathrm{~mm}$ CsI(Na) scintillator in the bottom of a similar $\mathrm{Nal}(\mathrm{TI})$ well detector. The Nal detector provides an active shield and/or active collimator for the $\mathrm{Cal}(\mathrm{Na})$ detector. Both are designed for low energy gammaemitters (i.e. I-129, Tc-99m, Se-75, etc.) or for Bremsstrahlung X-rays produced by pure beta emitters (such as P-32, Sr-90/Y-90, etc.). Targeted applications include the measurement of P-32 in tissue samples for biological research using the well detector and localizations of small amounts of low energy gamma emitters or pure beta emitters in the human body using the collimated detector. Results indicate low level limits of detection (LLD) for the well detector at $0.7 \mathrm{~Bq}$ for gamma emitters in the $20-100 \mathrm{keV}$ range and $11 \mathrm{~Bq}$ for $\mathrm{P}-32$ via Bremsstrahlung $\mathrm{X}$-ray generation (assuming a 60 second counting time). The collimated detector has LLDs of $11 \mathrm{~Bq}$ and $550 \mathrm{~Bq}$, respectively. ${ }^{13}$

Two more, large area detectors have been fabricated: one as a beta probe for environmental cleanup and decontamination surveys, and the second for measuring trace amounts of gamma-ray emitters or pure beta emitters over large areas of the body. The beta probe is being fabricated from a 2 $\mathrm{mm}$ by $127 \mathrm{~mm}$ diameter $\mathrm{CaF}_{2}$ scintillator coupled to a $127 \mathrm{~mm} \times 127 \mathrm{~mm} \mathrm{Nal}$ detector. The Nal acts as an active gamma-ray shield which can reduce the gamma-ray background in the beta detector to zero. This detector has not been tested to date. The second probe (utilizing $\mathrm{Csl}(\mathrm{Na})$ ) is initially targeted for measuring trace amounts of uranium (through the emission of $\sim 63 \mathrm{keV}$ and $\sim 93$ photons from ${ }^{234} \mathrm{Th}$ in the decay chain) in the body from either uptake of uranium (for example in fuel fabrication environments) or shrapnel from uranium tipped ammunition. ${ }^{14}$

\section{New Phoswich Design for Liquid Waste Stream Monitoring:}

A new detector design is in process for monitoring liquid waste streams from a radioactive waste processing system. MCNP Monte Carlo ${ }^{15}$ analysis has been used to determine operating parameters for 
this detector system and optimizes its design for measuring the trace amounts of alpha, beta and gamma-ray activity expected in effluent streams from nuclear waste cleanup processes. Simple MCNP $4 \mathrm{C}$ input files were constructed that modeled the three scintillation layers housed by an aluminum casing and surrounded by air using detector dimensions given below. The doping materials in the scintillators were not modeled in the MCNP environment, as their concentrations are low and can vary from different manufacturers.

While MCNP has several types of tallies to record the various interactions of radiation in system components, this report utilizes the F8, or pulse height, tally. This tally simulates gamma spectrometers for photons by returning probability distributions of energy deposition (or energy spectra) for a given set of energy bins. Separate F8 tallies were applied to each scintillator to model energy pulses that would be created in detectors by radiation interactions. Mode $p$, or transport of only photons, was used for all analysis other than cross-talk characteristics (i.e., mischaracterization of radiation events in the detector) and electron sources. In this mode, MCNP uses a full transport model for photon interactions but assumes that secondary electrons deposit all of their energy at the point of interaction. Mode $p$ proved to be an average of 250 times faster and nearly as accurate as Mode $p$ e, which performs full transport calculations for both photons and their secondary electrons. Mode $p$ e was used with beta source particles. Simulations typically modeled 10 million source photons or 500,000 source betas, which yielded relative errors in significant energy bins of $1 \%$ or less.

The previously designed, fabricated and tested phoswich detector for swipe counting ${ }^{12}$ consisted of a "linear" array with three scintillators (see Figure 1) placed on top of each other with a common diameter of $50.8 \mathrm{~mm}$ and viewed with a single photomultiplier tube. The scintillators $(\mathrm{ZnS}-0.0376 \mathrm{~mm}$, $\mathrm{CaF}_{2}-2.54 \mathrm{~mm}$ and $\mathrm{Nal}-25.4 \mathrm{~mm}$ ) interact preferentially with alpha, beta and gamma-ray radiation, respectively. This design allows preferential, but not exclusive, interaction of various radiations with specific layers. Taking into account and correcting for events that can occur in the "wrong" phosphor, this system was experimentally shown to have excellent capabilities (see Table 2) for properly identifying radiation coming from a mixed alpha/beta/gamma-ray source.

In an attempt to better understand this system and provide design guidance for a detector system to be used in monitoring effluents from nuclear waste treatment facilities, interactions in this detector were simulated (modeled) using MCNP. This analysis ${ }^{16}$ indicated that the thin $\mathrm{ZnS}$ layer adequately stops alpha particle energy, but reduces beta detection efficiency to essentially zero for beta $E_{\max }$ energies below $300 \mathrm{keV}$. The $\mathrm{CaF}_{2}$ layer, designed to keep any beta particle energy from entering the Nal detector results in an incorrect gamma-ray response that is approximately $23 \%$ of the Nal's response and is variable with energy. High energy beta events in the $\mathrm{CaF}_{2}$ can lead to Bremsstrahlung radiation being detected in the Nal. These errors must be corrected by system software.

To eliminate these shortcomings, a new co-axial geometry has been proposed to address some of the shortcomings identified above. ${ }^{17}$ This co-axial geometry has subsequently been optimized further for monitoring a liquid effluent stream that will nominally contain alpha, beta and gamma-ray radiation 
activities of $3.7 \times 10^{4} \mathrm{~Bq} / \mathrm{l}(10 \mathrm{nCi} / \mathrm{ml})$ each. $^{18,19}$ This geometry (see Figure 3) separates the $0.0244 \mathrm{~mm}$ thick alpha phosphor from the beta phosphor in a concentric circle with an inner radius of $10 \mathrm{~mm}$ and an outer radius of $50 \mathrm{~mm}$. The $\mathrm{CaF}_{2}$ is $1 \mathrm{~mm}$ thick with a radius of $10 \mathrm{~mm}$ inside of the alpha portion. $\mathrm{A}$ BGO detector (replacing the $\mathrm{Nal}$ ) also has a radius of $10 \mathrm{~mm}$, a height of $15 \mathrm{~mm}$ and is displaced from the surface of the detector by the $1 \mathrm{~mm}$ thick $\mathrm{CaF}_{2}$ and a $14 \mathrm{~mm}$ plastic "plug." All scintillators are backed by a common light pipe to effectively collect light output.

MCNP analysis of this design reveals that the ZnS detector's thickness no longer stops low energy beta particles from reaching the $\mathrm{CaF}_{2}$, thus eliminating the $300 \mathrm{keV} \mathrm{E}_{\max }$ threshold for beta particles seen in the previous "linear" detector. Also, since the relative number of alpha events reaching the detector will typically be small (due to their short range in aqueous effluent streams) the cross sectional area of the $\mathrm{ZnS}$ relative to the $\mathrm{CaF}_{2}$ has been greatly increased, increasing the alpha particle count rate. An estimate based upon average range for alpha and beta particles indicates that the ratio of beta to alpha particles reaching the detector would be about 30 to 1. For this design the area of $\mathrm{ZnS}$ in contact with the waste stream is $7500 \mathrm{~mm}^{2}$ vs $300 \mathrm{~mm}^{2}$ for the CaF2, or a ratio of 25 to 1 to compensate for this bias.

The $\mathrm{CaF}_{2}$ has been made thinner to reduce gamma-ray interactions. This thinner beta phosphor reduces its gamma-ray response from $23 \%$ in the previous, linear design to approximately $10 \%$, which can be more easily corrected by software.

The Nal detector was replaced by BGO due to its higher density and lower light output which more closely matches the light output of the $\mathrm{CaF}_{2}$, improving the dynamic range of the system. It is offset from the surface of the detector by $15 \mathrm{~mm}$ to reduce its efficiency for gamma-rays. This also reduces its sensitivity to X-rays produced throughout the detector by beta particles. The sensitivity of the BGO could be further reduced by making it even smaller and/or displacing it further from the surface of the waste stream, but the relative number of gamma events in the beta detector would increase to the point that software correction of these mis-characterized events would become problematic. For the $3.7 \times 10^{4} \mathrm{~Bq} / \mathrm{l}$ $(10 \mathrm{nCi} / \mathrm{ml})$ activity of alpha, beta and gamma-ray radiation assumed, the resulting count rates for a 100 $\mathrm{mm}$ diameter pipe would be $1000 \mathrm{cps}$ for gamma-rays and $30 \mathrm{cps}$ for both beta and alpha radiation.

\section{Conclusions:}

In conclusion, new phoswich detector designs have been proposed and modeled using MCNP, and several have been fabricated and tested. These detectors have been coupled to computer-based, digital signal processing systems to provide new features in flexibility and reliability. The phoswich designs improve on many of the limitations noted in previous designs and are optimized for a variety of radiation detection applications.

\section{Acknowledgements:}


This work has been funded by a DOE Health Physics Faculty Research Award (DOE DE FG02 92ER75704) and a DOE NEER grant (DOE DE FG07 01ID14118).

\section{References:}

1) R.B.OWEN, IRE Transactions on Nuclear Science, NS-9, (1962) 85.

2) "Harshaw TASC-12-A6 System Operation and Maintanence Manual - Automatic Alpha-Beta Counting System," Harshaw Chemical Company, Solon, Ohio (1979).

3) A.RUTTENBER, A.JAMES, Jr., K.KREISS, R.L.DOUGLAS, T.E.BUHL and J.MILLARD, Health Physics, 47 (1984) 21.

4) M.G.STRAUSS, A.T.KEANE and S.A.REINKE, IEEE Transactions on Nuclear Science, 37 (1990)

5) C.WANG, J.LEE and H.CHIOU, Applied Radiation and Isotopes, 45 (1994) 251.

6) S.USUDA, Nuclear Instruments and Methods, A340 (1995) 344.

7) M.TAKADA, S.TANIGUCHI, T.NAKAMURA and K.FUJITAKA, IEEE Transactions in Nuclear Science, A356 (1998) 334.

8) Al-Haddad, M. N., Lin, C., Miller, W. H., and Berliner, R. R., "Development and Testing of a Flash Analog-to-Digital Converter Based System for Pulse Shape Discrimination of Nuclear Radiation Pulses," IEEE Transactions on Nuclear Science, 41(5), p 1765-1779 (October 1994).

9) Gage Applied, Inc., a Subsidiary of Tektronix, http://www.gage-applied.com/.

10) Miller, W. H., Jackson, P., and Lin, C., "Utilization of a GaGe CompuScope 1012 computer-based oscilloscope for pulse shape discrimination and radiation spectroscopy," Nuclear Instruments \& Methods in Physics Research, Section A, 353(1-3) (December 30, 1994).

11) Miller, W. H., "An Alpha / Beta / Gamma Swipe Counter Based Upon Digital Pulse Shape Discrimination," Health Physics, Supplement to Vol. 72(6) ( June 1997).

12) White, T., and W.H. Miller, "A Triple-Crystal Phoswich Detector Using Digital Pulse Shape Discrimination for Alpha/Beta/Gamma Spectroscopy," Nucl. Instr. and Methods, A422, 144-147 (1999).

13) Pillai, S., J. Feng and W. H. Miller, "Phoswich Detectors with DSP Analysis for Low Energy, Low Activity Photon Measurements," Trans. Am. Nucl. Soc., 80, 64-65 (1999).

14) Duncan, L., "Applying the Phoswich Detector to Measuring Uranium in Workers," A Master's Degree Project, Nuclear Engineering Program, Missouri University (May, 1999).

15) Hendricks, J. S., "MCNP4C - Monte Carlo N-Particle Transport Code System," Distributed by Oak Ridge National Laboratory, RSICC Computer Code Collection, CCC-770 (July, 2000).

16) Childress, N. L. and W. H. Miller, "MCNP Analysis and Optimization of a Triple Crystal Phoswich Detector," Nuclear Instruments and Methods, Section A, 490(1-2), 263-270 (Sept 1, 2002).

17) Miller, W. H., N. L. Childress, "MCNP Phoswich Detector Optimization for Monitoring Processed Nuclear Waste Effluents," Trans. Am. Nucl. Soc., 86, 189 (June 10-13, 2002).

18) "Procurement Specifications: Salt Waste Processing Facility In-Line/On-Line Radionuclide Detection Monitor," J-SSP-H-00222, Revision A (June 2000).

19) Personal communication, Mark Amidon, Environmental Restoration and Engineering \& Technology, Savannah River Site (March 11, 2002). 
Table 1. Radiation type classification data for Harshaw system with analog and digital discrimination

\begin{tabular}{lrrrrrr} 
& \multicolumn{3}{c}{ Harshaw TASC-12 } & \multicolumn{3}{c}{ Digital System } \\
\hline \multicolumn{1}{c}{ Source } & \multicolumn{1}{c}{$\beta$} & \multicolumn{1}{c}{$\alpha$} & \multicolumn{1}{l}{$\gamma$} & \multicolumn{1}{l}{$\beta$} & \multicolumn{1}{l}{$\alpha$} & \multicolumn{1}{c}{$\gamma$} \\
\hline Beta (Tl-204) & $\mathbf{9 1 . 7 \%}$ & $1.1 \%$ & $7.1 \%$ & $\mathbf{8 8 . 9 \%}$ & $5.9 \%$ & $6.1 \%$ \\
Alpha (Pu-239) & $10.1 \%$ & $\mathbf{8 7 . 5 \%}$ & $2.4 \%$ & $3.2 \%$ & $\mathbf{9 4 . 3 \%}$ & $2.5 \%$ \\
Gamma (Cs-137) & $0.0 \%$ & $0.3 \%$ & $\mathbf{9 9 . 7 \%}$ & $0.1 \%$ & $0.1 \%$ & $\mathbf{9 9 . 8 \%}$ \\
\hline
\end{tabular}


Table 2. Radiation type classification data of various sources with digital system

\begin{tabular}{cccccc}
\hline Source & ${ }^{90} \mathrm{Sr}{ }^{90} \mathrm{Y}$ & ${ }^{204} \mathrm{Tl}$ & ${ }^{210} \mathrm{Po}$ & ${ }^{60} \mathrm{Co}$ & ${ }^{137} \mathrm{Cs}$ \\
\hline Beta & $\mathbf{9 9 . 1} \%$ & $\mathbf{9 4 . 8} \%$ & $0.0 \%$ & $2.8 \%$ & $0.5 \%$ \\
Alpha & $0.0 \%$ & $0.0 \%$ & $\mathbf{9 9 . 7} \%$ & $0.0 \%$ & $0.0 \%$ \\
Gamma & $0.9 \%$ & $5.2 \%$ & $0.3 \%$ & $\mathbf{9 7 . 2 \%}$ & $\mathbf{9 9 . 5 \%}$ \\
\hline
\end{tabular}


Figure 1: Three phosphor phoswich detector for simultaneous alpha, beta and gamma detection

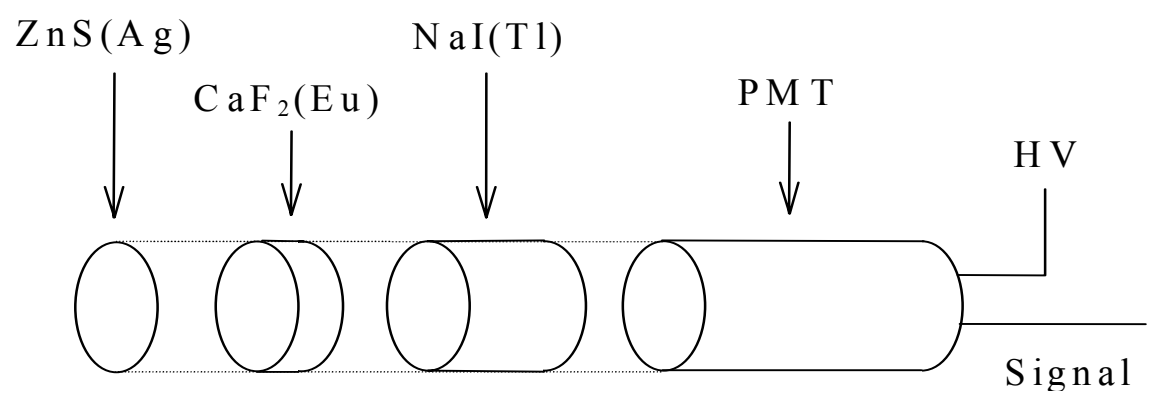


Figure 2: Digitally sampled pulses from the Harshaw $\mathrm{CsF}_{2} / \mathrm{Nal}$ phoswich TASC-12 detector

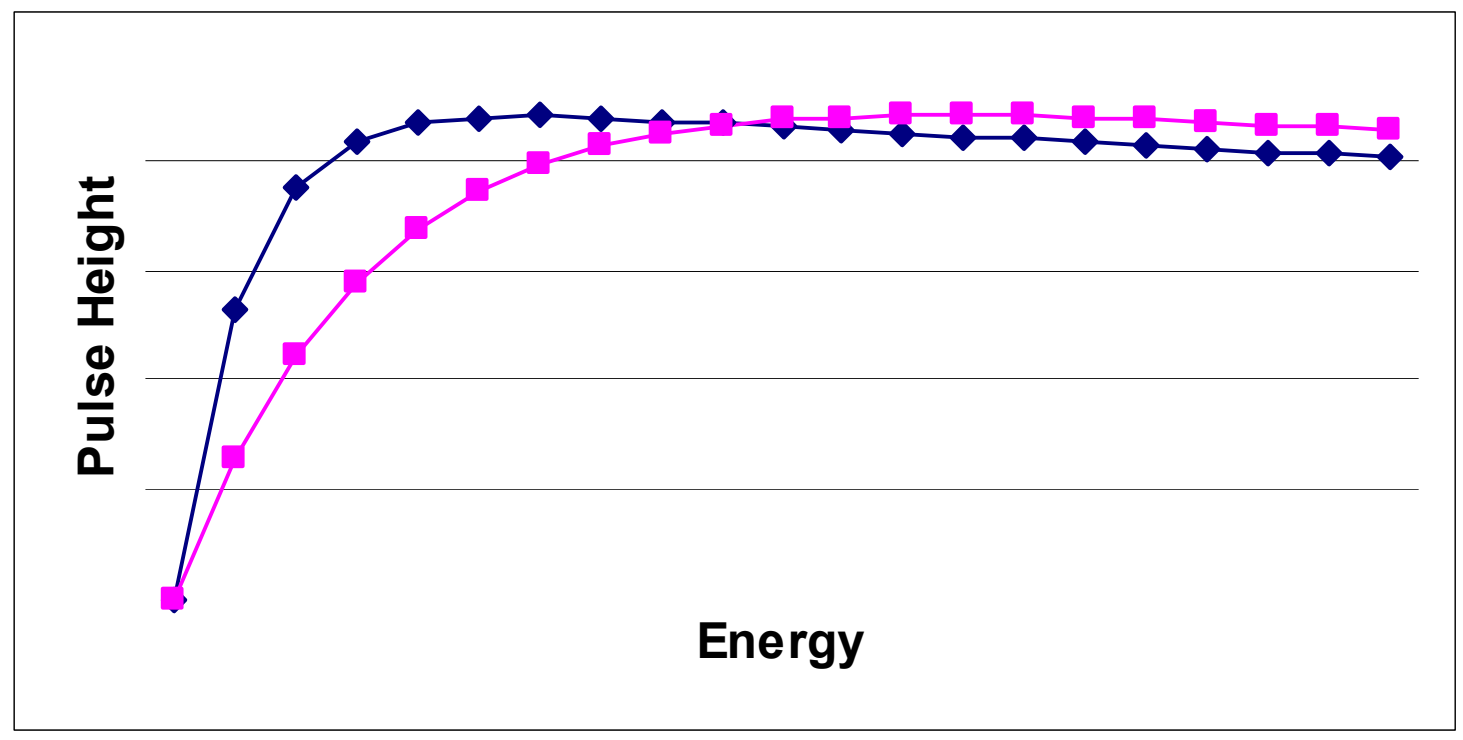


Figure 3: Optimized detector design for waste stream monitoring

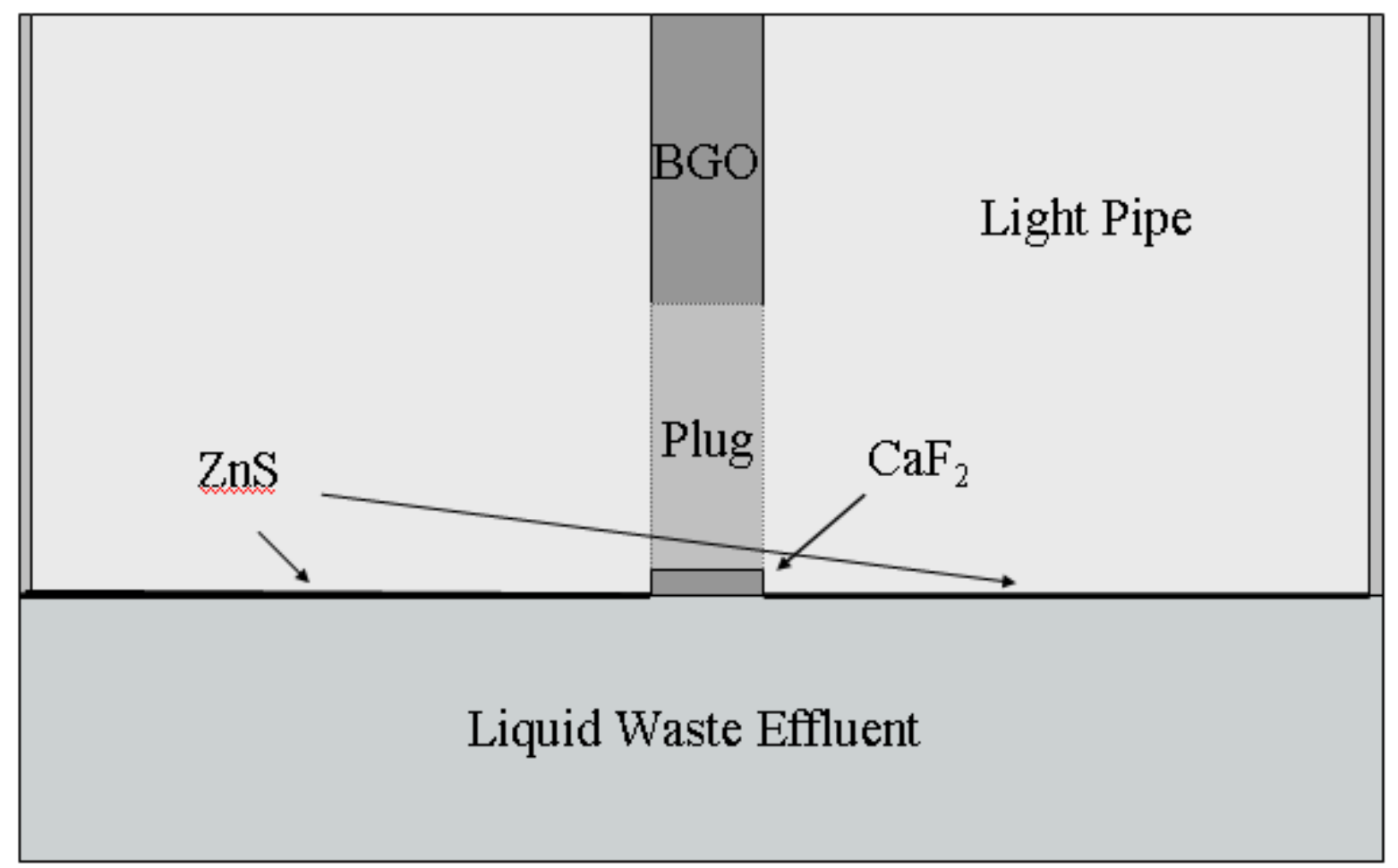

